\title{
Viscoelastic Properties of Cholesteric Liquid Crystals from Hydroxypropyl Cellulose Derivatives
}

\author{
Yuki Ogiwara ${ }^{1}$, Naoto Iwata ${ }^{1}$, and Seiichi Furumi ${ }^{{ }^{*}}$ \\ ${ }^{1}$ Department of Applied Chemistry, Faculty of Science, \\ Tokyo University of Science, 1-3 Kagurazaka, Shinjuku, Tokyo 162-8601, Japan \\ *furumi@rs.tus.ac.jp
}

\begin{abstract}
In this study, we investigated the rheological behavior of a cholesteric liquid crystalline polymer prepared by esterification of hydroxyl propyl cellulose (HPC) with propionyl chloride. The HPC ester tethering propionyl side chains (HPC-Pr) showed thermotropic cholesteric liquid crystal (CLC) phase with visible Bragg reflection in the temperature range between $100^{\circ} \mathrm{C}$ and $135^{\circ} \mathrm{C}$. Although visible Bragg reflection of HPC-Pr disappeared even after cooling from the temperature above isotropic phase transition temperature, we observed birefringence under crossed Nicols. Therefore, at this state, the molecular structure of HPCPr might be partially isotropic. The partially isotropic HPC-Pr had a relatively high viscosity as confirmed by rheological measurements. On the other hand, the viscosity decreased by shearing.

Keywords: Cellulose derivative, Cholesteric liquid crystals, Bragg reflection, Rheology, Viscoelasticity
\end{abstract}

\section{Introduction}

Cellulose is the most abundant polymer on the earth, and its derivatives often show liquid crystalline phase [1]. Especially, hydroxypropyl cellulose (HPC) is one of the most frequently investigated cellulose derivatives. For instance, HPC derivatives possessing acyl groups are known to show cholesteric liquid crystal (CLC) phase [24]. At the CLC phase, the chiral molecules are self-organized to form the helicoidal molecular structure with a periodic helical pitch. When unpolarized light propagates into the CLC, righthanded or left-handed circularly polarized light of a specific wavelength is reflected depending on the handedness of helical molecular structure. The specific reflection peak wavelength $\left(\lambda_{\text {ref }}\right)$ by CLC is quantitatively determined by the following formula:

$$
\lambda_{\text {ref }}=n p
$$

where $n$ is the average refractive index of CLC and $p$ is the helical pitch length [5]. Such a light reflection phenomenon can be regarded as a sort of Bragg reflection.
Previous report on the HPC derivatives with various acyl groups have shown that their average refractive indices are approximately 1.46 regardless of the alkyl chain length of their substitution groups [6]. By considering the experimental result, the reflection peak wavelength can be tuned by controlling the CLC helical pitch length by external stimuli. In the case of thermotropic CLCs, the helical pitch length is changed by temperature due to the thermodynamic motion of CLC molecules. Consequently, the reflection wavelength of CLC can be tuned by controlling the temperature [7].

The rheological behavior of HPC derivatives possessing acyl groups is interesting. Previous studies reported that sticky products are obtained by acylation of HPC $[3,4,8-10]$. This phenomenon is quite strange in that sticky polymer melts are produced from a powdery raw material of pristine HPC after the simple acylation. In addition, the fundamentals and applications of HPC derivatives with acyl groups are numerously discussed, focusing on their environmental-friendliness and CLC properties [10-15]. As well as the other CLC materials, it is expected to be applied for versatile 
photonic devices such as temperature sensors and reflective color displays. In the perspective of practical realization of them, the knowledge of their basic physical and mechanical properties is of prime importance. However, a limited number of studies on rheological behavior of acylated HPC derivatives have been reported, while there are many papers on that of HPC solutions. It is important to comprehend the rheological behavior of polymer melts.

In this report, we describe the optical and rheological properties of a HPC derivative possessing propionyl side chains (HPC-Pr), as shown in Fig. 1. The transmission spectral measurements of HPC-Pr revealed that the reflection peak wavelength is controlled by temperature, arising from the thermotropic CLC feature. Then, the viscoelasticity of HPC-Pr is measured to investigate the rheological behaviors caused by the helical molecular structure of CLC. The present report would pave the way to understand the intrinsic physical properties of HPC derivatives from both scientific and practical viewpoints.

\section{Experimental}

\subsection{Materials}

We used a commercially available substance of HPC (Fujifilm Wako Pure Chemical Co.; Viscosity of $2.0 \mathrm{wt} \%$ aqueous solution, $2.0 \sim 2.9 \mathrm{mPa} \cdot \mathrm{s})$. The number of hydroxypropyl groups per HPC monomer unit, i.e., molar substitution value $(M S)$, was determined to be 4.0 by using the ${ }^{1} \mathrm{H}-\mathrm{NMR}$ spectrum [16]. HPC was dried under vacuum before use. Anhydrous pyridine (Kanto Chemical Co., Inc.; 99.5\%), anhydrous acetone (Kanto Chemical Co., Inc.; 99.5\%), and propionyl chloride (Tokyo Chemical Industry Co. Ltd.; 98.0\%) were used as received.

Figure 1 shows the chemical structure of a HPC derivative possessing propionyl side chains (HPCPr). According to our previous report [14], HPCPr was synthesized by the esterification of HPC with propionyl chloride.

\subsection{Measurement of optical properties}

To fabricate a CLC cell, HPC-Pr was sandwiched between a pair of two glass plates with a gap of approximately $200 \mu \mathrm{m}$. Transmission spectra of the CLC cell were measured using a compact CCD spectrometer (Ocean Optics, USB2000+) equipped with a tungsten halogen light source for the probing white light. The temperature of CLC cell was precisely controlled using a hot stage system (Mettler Toledo, HS82 and HS1).

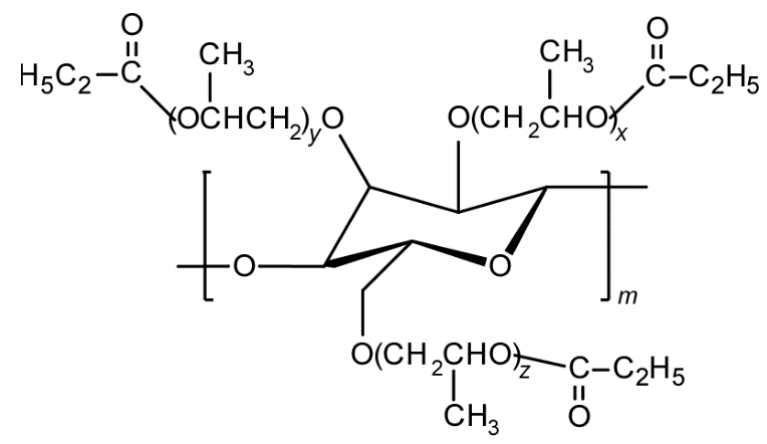

Fig. 1. Chemical structure of a hydroxypropyl cellulose derivative possessing propionyl side chains (HPC-Pr).

\subsection{Measurement of rheological properties}

The viscosity measurements were performed using a stress-controlled rheometer (Anton Paar, MCR102) equipped with a stainless-steel parallel plate with a diameter of $25 \mathrm{~mm}$. HPC-Pr was sandwiched at the gap of approximately $500 \mu \mathrm{m}$. In this experiment, the shear rate $(\dot{\gamma})$ was increased from $0.01 \mathrm{~s}^{-1}$ to $100 \mathrm{~s}^{-1}$ and then decreased from 100 $\mathrm{s}^{-1}$ to $0.01 \mathrm{~s}^{-1}$ taking $41 \mathrm{sec}$ in each way. By using a forced convection oven, the temperature of HPC$\operatorname{Pr}$ was maintained at $100{ }^{\circ} \mathrm{C}$ during the measurements. As heated at this temperature of $100{ }^{\circ} \mathrm{C}$, a reflection peak of HPC-Pr emerged around $400 \mathrm{~nm}$, as mentioned below.

Oscillatory shear rheology measurements were also conducted by using the above-mentioned rheometer. The storage modulus $\left(G^{\prime}\right)$ and loss modulus $\left(G^{\prime}\right)$ were measured at angular frequencies $(\omega)$ ranging from $100 \mathrm{rad} / \mathrm{s}$ to $0.1 \mathrm{rad} / \mathrm{s}$ at the temperature between $40^{\circ} \mathrm{C}$ to $100^{\circ} \mathrm{C}$ at the intervals of $20^{\circ} \mathrm{C}$. The strain amplitude was adjusted in the range of $0.2 \%$ to $2.0 \%$, which was sufficiently small to measure the linear viscoelasticity. The master curves were created by shifting the $G$ ' and $G$ "' results in logarithmic scales according to the timetemperature superposition principle with reference temperature $\left(T_{\text {ref }}\right)$ of $100{ }^{\circ} \mathrm{C}$. When shifting the results, the amounts of horizontal and vertical shifts of the curves at each temperature are called the horizontal shift factor $\left(a_{\mathrm{T}}\right)$ and vertical shift factor $\left(b_{\mathrm{T}}\right)$, respectively. The values $a_{\mathrm{T}}$ and $b_{\mathrm{T}}$ are dependent on the temperature.

Before each measurement, HPC-Pr was either preheated or presheared to erase any remaining the historical molecular orientation. Hereafter, the samples are noted as $\mathrm{Pr}-\mathrm{H}$ or Pr-S, respectively. $\mathrm{Pr}-\mathrm{H}$ was prepared by heating at $155^{\circ} \mathrm{C}$ for $5 \mathrm{~min}$ in 
the rheometer, which was higher than the isotropic phase transition temperature of HPC-Pr $\left(T_{\mathrm{i}}=150\right.$ ${ }^{\circ} \mathrm{C}$ ) to ensure the random orientation of cholesteric texture confirmed by polarized optical microscopic observation. Pr-S was prepared by shearing at the rate of $10 \mathrm{~s}^{-1}$ for $200 \mathrm{sec}$ heated at $100{ }^{\circ} \mathrm{C}$, which was sufficiently long enough to reach its steady state of molecular alignment.

\section{Results and discussion}

\subsection{Synthesis of HPC-Pr}

We confirmed that all hydroxyl groups in the side chains of HPC are completely substituted with propionyl groups to form HPC-Pr. According to the identification method described in our previous report [14], the ${ }^{1} \mathrm{H}-\mathrm{NMR}$ spectrum of HPC-Pr in $\mathrm{CDCl}_{3}$ indicated that all hydroxyl groups of HPC are esterified by propionyl chloride. Also, the FTIR spectrum showed the disappearance of a broad peak around $3500 \mathrm{~cm}^{-1}$, assigned to the $\mathrm{O}-\mathrm{H}$ stretching vibration of pristine HPC, after the esterification of HPC with propionyl chloride.

\subsection{Reflection properties of HPC-Pr}

The Bragg reflection peak wavelength of HPC-Pr was dependent on the temperature. Figure 2 shows the changes in the transmission spectrum of a cell of HPC-Pr upon stepwise heating from $100^{\circ} \mathrm{C}$ to $135{ }^{\circ} \mathrm{C}$ at the intervals of $5{ }^{\circ} \mathrm{C}$. The Bragg reflection peak was continuously red-shifted from $400 \mathrm{~nm}$ to $635 \mathrm{~nm}$ upon heating from $100{ }^{\circ} \mathrm{C}$ to 135 ${ }^{\circ} \mathrm{C}$. Such a shift of Bragg reflection peak can be attributed to the increase of the helical pitch length of the thermotropic CLC induced by temperature $[7,17]$. Furthermore, we confirmed that the

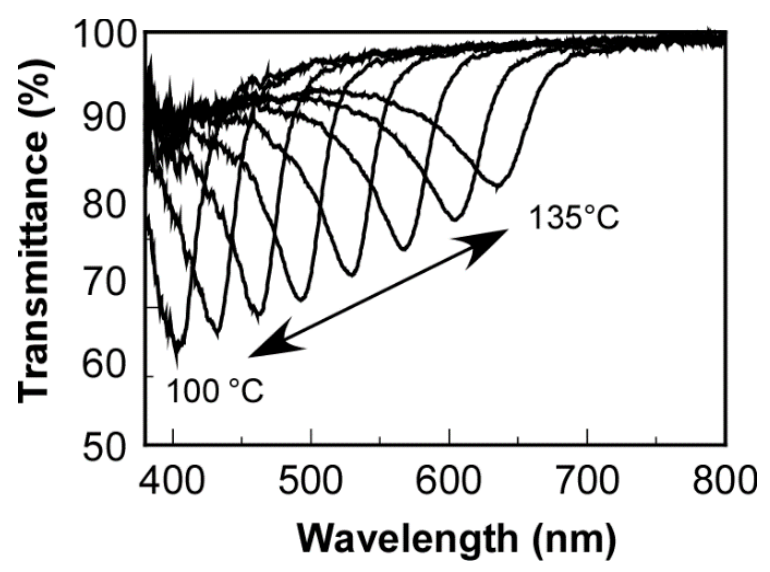

Fig. 2. Transmission spectral changes in the CLC cell of HPC-Pr upon stepwise heating from $100{ }^{\circ} \mathrm{C}$ to 135 ${ }^{\circ} \mathrm{C}$ at the intervals of $5{ }^{\circ} \mathrm{C}$. The reflection peak was red-shifted, accompanied by the spectral broadening, upon the heating process. reflection peak is blue-shifted from $635 \mathrm{~nm}$ to 400 $\mathrm{nm}$ upon cooling process from $135^{\circ} \mathrm{C}$ to $100^{\circ} \mathrm{C}$. Therefore, the reflection wavelength shift by temperature was found to be fully reversible in the range between $100{ }^{\circ} \mathrm{C}$ and $135^{\circ} \mathrm{C}$.

\subsection{Pretreatment of HPC-Pr before rheological measurements [18]}

Figure 2 also shows the broadening of reflection band as well as the decrease of reflection intensity by elevating the temperature from $100^{\circ} \mathrm{C}$ to $135^{\circ} \mathrm{C}$, suggesting that the helical axis of CLC gradually becomes the disordered by heating close to the isotropic phase temperature. This is also supported by the fact that the Bragg reflection is not observed at $100{ }^{\circ} \mathrm{C}$ for at least $3 \mathrm{~h}$ after heating at $155^{\circ} \mathrm{C}$ for $5 \mathrm{~min}$ once. This result emphasizes that the preheating process deteriorates the orientational order of helical axis of CLC. On the other hand, as HPC-Pr was heated at $155{ }^{\circ} \mathrm{C}$, followed by 100 ${ }^{\circ} \mathrm{C}$, the polarized optical microscopic observation between crossed Nicols confirmed that HPC-Pr shows slight birefringence. However, at this stage, no Bragg reflection color was observed. Taking the overall facts in account, the molecular structure of HPC-Pr after preheating process can be interpreted as follows. Although the CLC molecules form the helicoidal structure at the microscopic scale such as one helical pitch or less, the helical axis is aligned in random directions. As a result, Bragg reflection was not observed due to the randomly-aligned helical axis of CLC structure while the birefringence was observed due to the formation of CLC structure at microscopic scale. This motivated us to investigate the shearing effect of HPC-Pr.

The preshearing time was determined to be 200

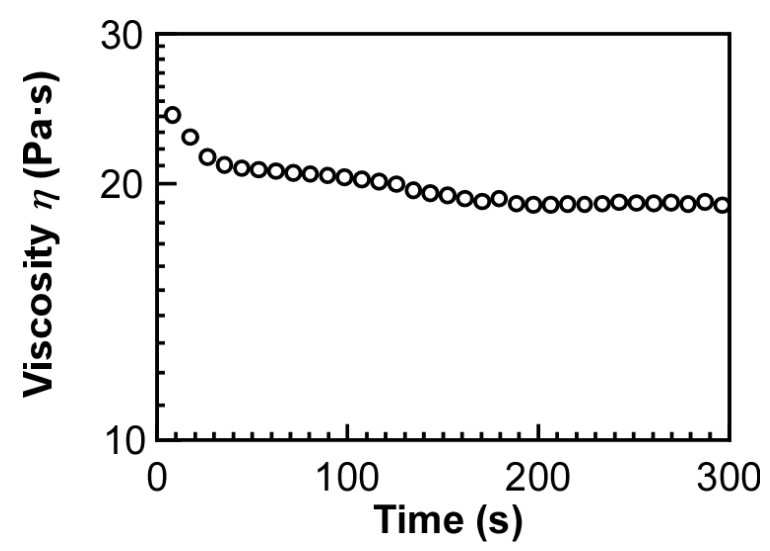

Fig. 3. Time dependence of the viscosity $(\eta)(\dot{\gamma}=10$ $\mathrm{s}^{-1}, 100{ }^{\circ} \mathrm{C}$ ) of a preheated CLC of HPC-Pr, which reached the steady state by shearing at $200 \mathrm{sec}$. 
sec, which is sufficient to align CLC layers. Figure 3 shows the time dependence of viscosity of HPC-Pr measured at $100{ }^{\circ} \mathrm{C}$ by shearing at a constant rate of $10 \mathrm{~s}^{-1}$. The viscosity gradually decreased with taking the measurement time up to $150 \mathrm{sec}$. However, the viscosity became constant at $19 \mathrm{~Pa} \cdot \mathrm{s}$ despite the prolonged measurement time of $150 \mathrm{sec}$ or more, suggesting that the sample reached its steady state. This experimental result also implies that the alignment of CLC layers is completed by shearing at $10 \mathrm{~s}^{-1}$ for $150 \mathrm{sec}$.

\subsection{Rheological properties of HPC-Pr}

Although both Pr-H and Pr-S exhibited shear thinning behavior, their flow curves were different when $\dot{\gamma}$ is increased, as shown in Fig. 4. Shear thinning is a decrease of viscosity with increasing the shear rate, and often occurred by structure deformation of the sample [19]. This indicates that the CLC structure of HPC-Pr is changed by shearing. Thereby, we focus on the difference of viscosity between Pr-H and Pr-S. It is well-known that the viscosity is crucially influenced by the molecular arrangements.

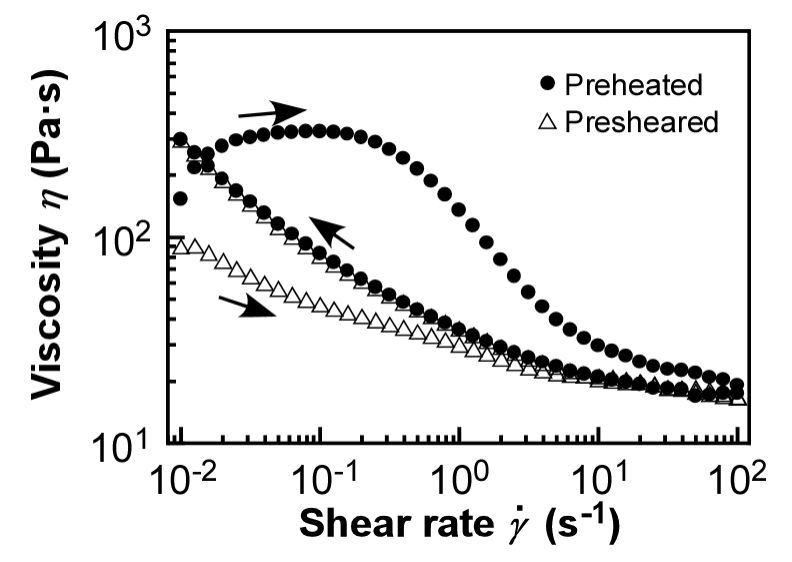

Fig. 4. Shear-rate dependences at $100{ }^{\circ} \mathrm{C}$ of the apparent viscosity of $\mathrm{Pr}-\mathrm{H}$ and $\mathrm{Pr}-\mathrm{S}$ samples. The value $\eta$ of Pr-H was higher than that of Pr-S in the increasing $\dot{\gamma}$ process, whereas they showed the same profiles in the decreasing $\dot{\gamma}$ process.

For instance, the viscosity of polymers whose chains entangle themselves tends to be higher than that of unentangled one [20]. The viscosity of Pr$\mathrm{H}$ at $0.2 \mathrm{~s}^{-1}$ was $3.0 \times 10^{2} \mathrm{~Pa} \cdot \mathrm{s}$, which was up to 7.5 times higher than that of the Pr-S (40 Pa.s). Such high viscosity of $\mathrm{Pr}-\mathrm{H}$ can be attributed to the randomly oriented CLC layers. When the Pr-H is sheared, the additional force is requisite to generate well alignment of CLC layers from random state [18].
On the other hand, when $\dot{\gamma}$ was decreased, the viscosity of $\mathrm{Pr}-\mathrm{H}$ and $\mathrm{Pr}-\mathrm{S}$ were almost identical regardless of $\dot{\gamma}$. This is because the CLC alignment is completed in the process of increasing $\dot{\gamma}$ for both Pr-H and Pr-S.

The flow curve of Pr-H also exhibited shear thickening behavior in the $\dot{\gamma}$ range from $0.01 \mathrm{~s}^{-1}$ to $0.1 \mathrm{~s}^{-1}$. In general, the shear thickening behavior appears with structure formation by shear. This fact implies the alignment of CLC layers is induced when $\dot{\gamma}$ is below $0.01 \mathrm{~s}^{-1}$, which is reasonable for considering the randomly-arranged CLC layers. $\mathrm{Pr}-\mathrm{H}$ seems to be more viscous than Pr-S.

Figures 5 (a) and 5 (b) shows the master curves of $G^{\prime}$ and $G^{\prime \prime}$ of Pr-H and Pr-S heated at $T_{\text {ref }}$ of 100 ${ }^{\circ} \mathrm{C}$. The master curve of Pr-H given in Fig. 5 (a) can be divided into two regions by the difference of relationship between $G^{\prime}$ and $G^{\prime \prime}$. In the region of $\omega$ below $4.0 \times 10^{-1} \mathrm{rad} / \mathrm{s}, G^{\prime}$ exceeds $G$ ' and reaches a plateau, which implies that $\mathrm{Pr}-\mathrm{H}$ is more solid-like in this region. This rigidity of $\mathrm{Pr}-\mathrm{H}$ can be caused by the randomly oriented CLC layers as described above. At higher region of $\omega$ over $4.0 \times 10^{-1} \mathrm{rad} / \mathrm{s}$, $G$ ' became larger than $G$ ' and the slopes of $G$ ' and $G$ ' in the range above $\omega=1.0 \times 10^{2} \mathrm{rad} / \mathrm{s}$ were
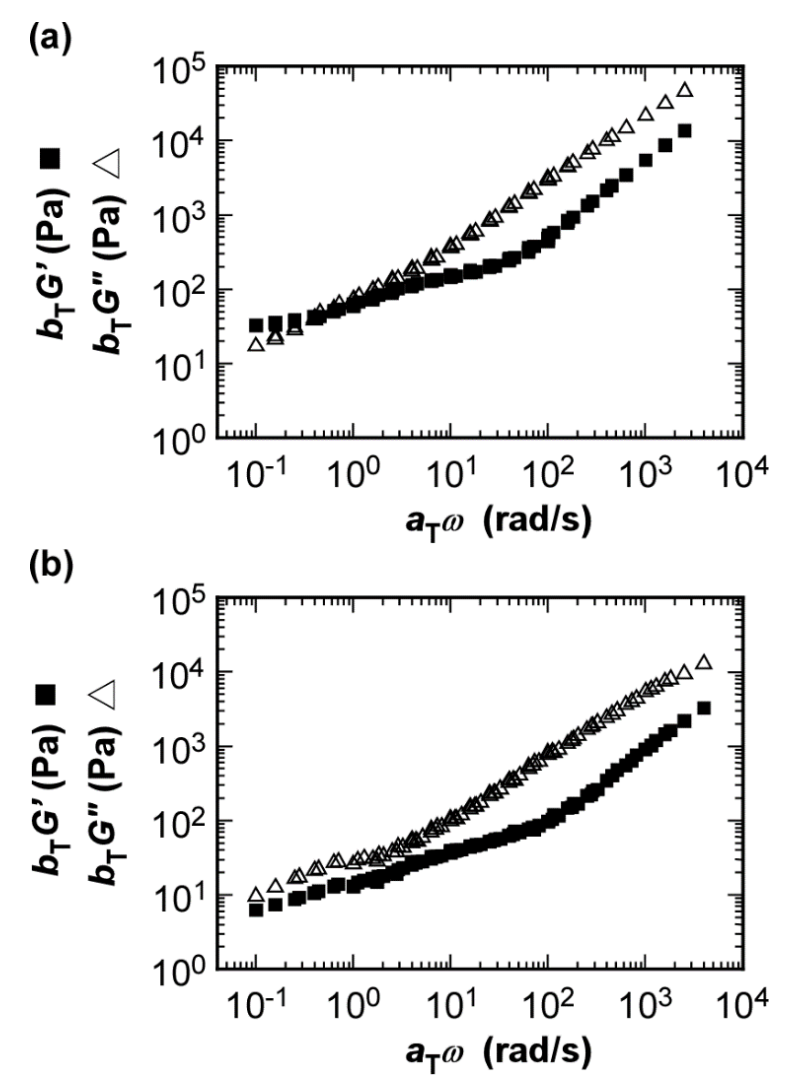

Fig. 5. Master curves of the storage modulus $\left(G^{\prime}\right)$ and loss modulus ( $G$ ') of HPC-Pr heated at $T_{\text {ref }}$ of $100{ }^{\circ} \mathrm{C}$ (a) The preheated sample $(\mathrm{Pr}-\mathrm{H}) . \quad$ (b) The presheared
sample (Pr-S). 
estimated to be approximately 1.0 and 0.8 , respectively. These values are different from those of the ideal Newtonian fluid, which would be 2.0 and 1.0, respectively. Such smaller $\omega$ dependences of $G^{\prime}$ and $G^{\prime \prime}$ can be attributed to the disturbed flow behavior caused by the molecular orientation of CLCs.

The master curve of Pr-S presented in Fig. 5 (b) is reasonably characterized by two differences when compared with that of Pr-H as follows. First, $G$ ' exceeded $G^{\prime}$ in the entire $\omega$ range, and no plateau of $G$ ' was observed. This indicates that Pr-S is more liquid-like in lower $\omega$ regions. This liquid-like behavior might be happened by the aligned CLC structure. Second, both $G$ ' and $G$ ' of Pr-H were higher than those of Pr-S in the entire $\omega$ range. On the other hand, the slopes of both $G^{\prime}$ and $G^{\prime \prime}$ in the range above $\omega=1.0 \times 10^{2} \mathrm{rad} / \mathrm{s}$ were approximately 1.0 and 0.8 , respectively, which was the same as those of Pr-H.

Considering the overall results, it turned out that $\mathrm{Pr}-\mathrm{H}$ is more viscous and rigid rather than $\mathrm{Pr}-\mathrm{S}$. This rigidity of Pr-H might stem from the randomly oriented CLC layers, as mentioned above.

\section{Conclusion}

In this study, the rheological behavior of a CLC polymer derived from HPC was investigated. As the CLC polymer was preheated above the isotropic phase transition temperature, the partially isotropic structure remained even after cooling. This was confirmed by the fact that the color derived from Bragg reflection did not appear but birefringence appears in this state. In this way, the preheated CLC polymers behave more viscously and rigidly than the presheared ones, which might be attributed to their randomly oriented CLC layers. The experimental evidence is expected to be available to apply the general CLC polymers for not only the comprehension of physical properties from a scientific standpoint, but also the fabrication of novel photonic devices with CLC materials from a practical standpoint [21-25].

\section{Acknowledgements}

All of the authors express sincere thanks to Mr. R. Kawanishi of Anton Paar Japan K. K. for his technical supports and advices on the rheological measurements. Moreover, the authors would like to acknowledge Messrs. K. Hayata, Shimokawa, and Baba and Mses. R. Aoki, A. Kawaguchi, Furukawa, and S. Saito for their helpful discussions. One of the authors (S.F.) is deeply indebted to the financial supports from the Grant-in-Aid for Scientific Research (B) (No. 17H03848 and No. 21H02261) of the Ministry of Education, Science, Sports and Culture (MEXT) of Japan and NEXCO Group Companies' Support Fund to Disaster Prevention Measures on Expressways.

\section{References}

1. D. G. Gray, J. Appl. Polym. Sci.: Appl. Polym. Symp., 37 (1983) 179.

2. S.-L. Tseng, A. Valente, and D. G. Gray, Macromolecules, 14 (1981) 715.

3. S. L. Tseng, G. V Laivins, and D. G. Gray, Macromolecules, 15 (1982) 1262.

4. S. N. Bhadani and D. G. Gray, Mol. Cryst. Liq. Cryst., 99 (1983) 29.

5. H. de Vries, Acta Crystallogr., 4 (1951) 219.

6. B. Huang, J. J. Ge, Y. Li, and H. Hou, Polymer, 48 (2007) 264.

7. H. Kosho, S. Hiramatsu, T. Nishi, Y. Tanaka, S. Kawauchi, and J. Watanabe, High Perform. Polym., 11 (1999) 41.

8. I. Rusig, J. Dedier, C. Filliatre, M. H. Godhino, L. Varichon, and P. Sixou, J. Polym. Sci. Part A Polym. Chem., 30 (1992) 895.

9. K. Hayata, T. Suzuki, M. Fukawa, and S. Furumi, J. Photopolym. Sci. Technol., 32 (2019) 645.

10. M. Fukawa, A. Kawaguchi, K. Hayata, R. Aoki, M. Furukawa, and S. Furumi, J. Photopolym. Sci. Technol., 32 (2019) 633.

11. R. Aoki, M. Fukawa, and S. Furumi, J. Photopolym. Sci. Technol., 32 (2019) 651.

12. M. Fukawa, K. Suzuki, and S. Furumi, J. Photopolym. Sci. Technol., 31 (2018) 563.

13. N. Tamaoki, Adv. Mater., 13 (2001) 1135.

14. T. Ishizaki, S. Uenuma, and S. Furumi, Kobunshi Ronbunshu, 72 (2015) 737 (in Japanese).

15. H. Shimokawa, K. Hayata, M. Fukawa, and S. Furumi, J. Photopolym. Sci. Technol., 33 (2020) 467.

16. F. F. L. Ho, R. R. Kohler, and G. A. Ward, Anal. Chem., 44 (1972) 178.

17. G. V. Laivins and D. G. Gray, Polymer., 26 (1985) 1435.

18. L. Ramos, M. Zapotocky, T. C. Lubensky, and D. A. Weitz, Phys. Rev. E, 66 (2002) 031711.

19. W. W. Graessley, J. Chem. Phys., 47 (1967) 5057.

20. M. Doi and S. F. Edwards, "The Theory of Polymer Dynamics", Clareodon Press, Oxford, 1986, p. 230-236. 
21. S. Furumi, S. Yokoyama, A. Otomo, and S. Mashiko, Appl. Phys. Lett., 82 (2003) 16.

22. S. Furumi, S. Yokoyama, A. Otomo, and S. Mashiko, Appl. Phys. Lett., 84 (2004) 2491.
23. S. Furumi and N. Tamaoki, Adv. Mater, 22 (2010) 886.

24. S. Furumi, Chem. Rec., 10 (2010) 394.

25. S. Furumi, Polym. J., 45 (2013) 579. 